Rev.MVZ Córdoba 17(2):3087-3091, 2012.

CASO CLÍNICO

\title{
Plasmocitoma extramedular de nódulos linfáticos en un perro
}

\section{Lymph nodes extramedullary plasmacytoma in a dog}

\author{
Juan Ballut $\mathrm{P}_{1}{ }^{1 *} \mathrm{M} . \mathrm{Sc}$, Lissy Padrón $\mathrm{C}_{1}{ }^{2 \dagger} \mathrm{MVZ}$.
}

\begin{abstract}
${ }^{1}$ Universidad de Córdoba, Facultad de Medicina Veterinaria y Zootecnia. Departamento de Ciencias Pecuarias. Montería, Córdoba, Colombia. ${ }^{2}$ Centro Veterinario Zoo'gar Montería, Córdoba, Colombia. ${ }^{\dagger Q}$. E. P. D. *Correspondencia: jcballut@sinu.unicordoba.edu.co
\end{abstract}

Recibido: Julio de 2010; Aceptado: Diciembre de 2011.

\section{RESUMEN}

El plasmocitoma extramedular es una masa tumoral constituida por células plasmáticas distantes de la médula ósea, clasificándose como tumor cutáneo y no es de presentación frecuente en perros. Se atendió en la en el centro veterinario ZOOGAR un paciente canino macho, mestizo, de ocho años de edad con evidente linfadenopatía generalizada al examen clínico. Al examen histológico post mortem, reveló células plasmáticas en nódulos linfáticos, encontrándose focos de necrosis, coagulación y hemorragia tanto en la parte cortical como la medular; con remodelación tisular por tejido conectivo. El hígado y bazo presentaron evidencia de metástasis, específicamente el hígado, a nivel de las triadas portales y áreas centrolobulillares. Los plasmocitomas extramedulares de nódulos linfáticos son raros en perros y no ha sido reportado en estos órganos.

Palabras clave: Extramedular, nódulos linfáticos, plasmocitoma (Fuente:DeSC).

\begin{abstract}
Extramedullary plasmacytoma is a tumor mass composed of plasma cells distant from the bone marrow, classified as skin tumor and is not often present in dogs. The veterinary center ZOOGAR received a canine patient, male, mixed breed; age eight with generalized lymphadenopathy evident after clinical examination. Postmortem histological examination revealed plasmatic cells in lymph nodes, finding sources of necrosis, coagulation and hemorrhage in both the cortical and medullary part; with tissue remodeling by connective tissue. The liver and spleen showed evidence of metastasis, specifically the liver, at the level of portal triads and centrilobular areas. The lymph node extramedullary plasmacytomas are rare in dogs and have not yet been reported in these organs.
\end{abstract}

Key words: Extramedullary, Lymph nodes, plasmacytoma (Source:DeSC). 


\section{INTRODUCCIÓN}

El plasmocitoma extramedular, es una masa tumoral constituida por células plasmáticas distante de la medula ósea (1). Según clasificación de tumores cutáneos, este se encuentra dentro del grupo de neoplasias de células redondas (2). Los plasmocitomas son de presentación frecuente en perros; aunque es usual en perros viejos, mayores de 9 años de edad. Los plasmocitomas extramedulares, comúnmente se encuentran localizados en zonas mucocutáneas y la mayoría de los casos son tumores únicos (3). Otros sitios menos comunes reportados de plasmocitoma extramedular incluyen, útero (4), tráquea (5), laringe (6), tracto gastrointestinal $(7,8)$ e intraocular (9).

El diagnóstico generalmente es por histopatología e inmunohistoquímica $(10,11)$. Los plasmocitomas por inmunohistoquímica, se caracterizan por la presencia de inmunoglobulinas tipo lambda monoclonal de cadenas livianas (12). Uno de los diagnósticos diferenciales del plasmocitoma extramedular, es el mieloma múltiple; se diferencian en base a una combinación de pruebas histológicas especificas y un estudio de los sistemas afectados $(13,14)$.

La terapéutica empleada en los plasmocitomas extramedulares, es la radioterapia, la escisión quirúrgica, quimioterapia o cualquier combinación de estas $(2,5,6)$.

Los plasmocitomas extramedulares, a diferencia del mieloma múltiple, generalmente tienen un pronóstico favorable, especialmente aquellos únicos o solitarios $(15,16)$.

Historia clínica. En una clínica Veterinaria privada de la ciudad de Montería-Colombia fue atendido un canino macho, sin raza definida, de ocho años de edad. El motivo de la consulta era inapetencia, disnea y pérdida progresiva de masa corporal.
Examen físico. El examen físico reveló los siguientes hallazgos:

Frecuencia cardiaca (124 latidos por minuto), pulso (124 pulsaciones por minuto, fuerte y regular), temperatura corporal $\left(37.9^{\circ} \mathrm{C}\right)$, peso corporal (24 kilogramos), condición corporal (delgado), distención abdominal, disnea espiratoria, murmullo vesicular disminuido, nódulos linfáticos palpables aumentados de tamaño, presencia de edema frio subcutáneo en miembro posterior izquierdo acompañado de claudicación; a la palpación abdominal, se evidenció hepatomegalia, esplenomegalia y prueba de macicez horizontal positiva.

Exámenes complementarios. Al quinto día posterior a la primera consulta, el animal llegó a control y se tomaron muestras de sangre y orina para realización de exámenes como hemoleucograma y urianalisis.

Hemoleucograma. Presentó anemia leve, normocítica normocrómica, leucocitosis moderada con neutrofília y desviación a la izquierda degenerativa (Tabla 1).

Urianálisis. El único hallazgo de importancia, correspondió a bilirrubinuria (reacción de $3+$ ) asociada a una densidad urinaria dentro los rangos referenciales (1.025).

Manejo clínico y evolución. Como acercamiento terapéutico inicial, se instauró tratamiento antibiótico y antiinflamatorio con un bacteriostático de amplio espectro (doxiciclina: $10 \mathrm{mg} / \mathrm{kg} /$ día VO) y un corticosteroide (prednisolona: $0.25 \mathrm{mg} /$ $\mathrm{kg} /$ día VO) respectivamente, sospechándose de un trastorno inflamatorio de tipo infeccioso de las vías respiratorias bajas. Trascurrido cinco días, el paciente no respondió favorablemente al tratamiento inicial, motivo por el cual se suspendió el tratamiento antimicrobiano y se incorporó al tratamiento un diurético de asa (furosemida: $2 \mathrm{mg} / \mathrm{kg} / 12$ horas VO) y acido orótico con oxipurina, como un manejo terapéutico de una hipoproteinemia por insuficiencia hepática, debido al síndrome ascítico y la presencia de bilirrubinuria.

Tabla1. Resultados del hemoleucograma al quinto día.

\begin{tabular}{|c|c|c|c|c|c|c|c|}
\hline Analito & Muestra & V. REF & Analito & Muestra & V. REF & Muestra & V. REF \\
\hline Hematocrito & $28 \%$ & $37-48$ & Leucocitos & & & $22.5 \mathrm{mil} / \mathrm{ul}$ & $8.000-14.000$ \\
\hline Hemoglobina & $9.5 \mathrm{~g} / \mathrm{dl}$ & $12-15$ & Neutrófilos & $65 \%$ & $55-75$ & $14.625 \mathrm{ul}$ & $3.300-10.000$ \\
\hline Eritrocitos & $4.56 \mathrm{mill} / \mathrm{ul}$ & $5.5-8.5$ & Linfocitos & $15 \%$ & $12-30$ & $3.375 \mathrm{ul}$ & $1.000-4.500$ \\
\hline VCM & $61.4 \mathrm{fl}$ & $60-77$ & Monocitos & --- & $1-7$ & & $<700$ \\
\hline C.H.C.M & $33.9 \mathrm{~g} / \mathrm{dl}$ & $32-37$ & Banda & $20 \%$ & 0 & $4.500 \mathrm{ul}$ & 0 \\
\hline Reticulocitos & $1.5 \%$ & $1-1.3$ & Eosinófilos & ---- & $0-5$ & & $100-1.500$ \\
\hline Plaquetas & 200.000 & $200-400$ & & & & & \\
\hline
\end{tabular}


El animal muere a los cuatro meses postratamiento, sin presentar mejoría evidente.

Examen Necroscópico. Los hallazgos más relevantes en la necropsia fueron: aumento de tamaño de los nódulos linfáticos externos (mandibulares, preescapulares, inguinales y poplíteos), además de los internos como los mediastínicos y mesentéricos; esplenomegalia, hepatomegalia; efusión pleural y pericárdica.

Examen Histopatológico. Se obtuvo muestras de nódulos linfáticos, bazo, hígado, riñón y pulmón; fueron conservadas en formalina al $10 \%$ y procesadas en un laboratorio médico veterinario en la ciudad de Bogotá - Colombia. Los hallazgos histopatológicos fueron:

Las muestras de pulmón, no se encontraron hallazgos de neoplasias.

En nódulos linfáticos se observó presencia de células redondas con núcleos redondeados, algunos con carátula de reloj, cromatina grumosa y nucléolos evidentes. En la mayoría de la población celular, el núcleo está desplazado hacia un extremo del citoplasma. Índice mitótico bajo y la diferenciación celular era definida (Figuras 1a y 1b).

El bazo y el hígado presentaron evidencia de metastásis; en el bazo se encontró, focos de necrosis de coagulación y hemorragia
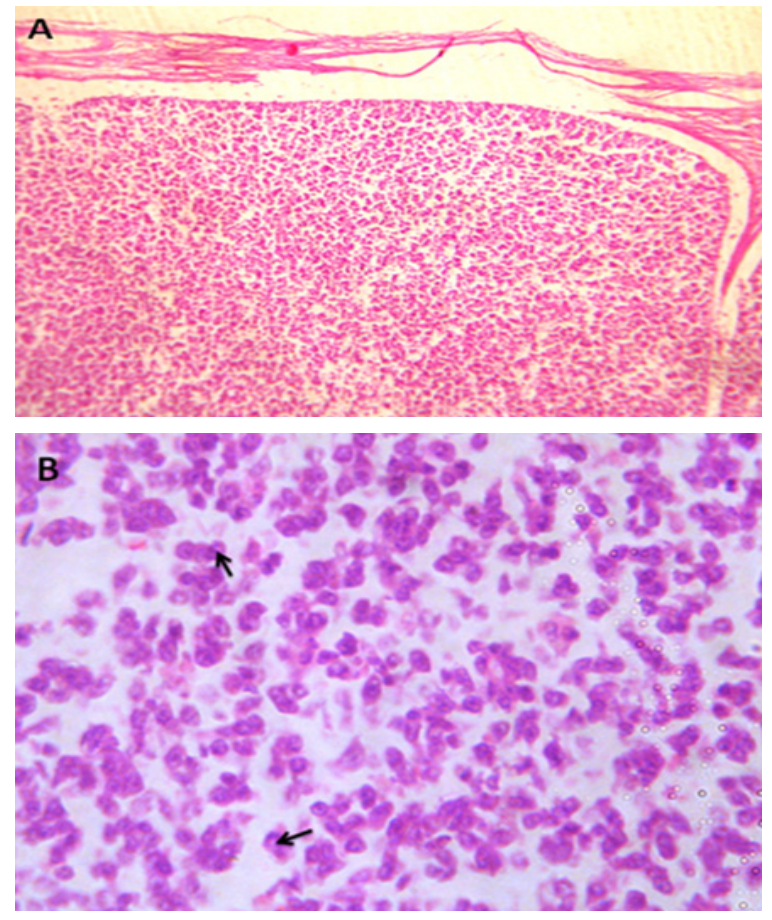

Figura 1. Corte histológico de un nódulo linfático: (A) infiltración de plasmocitos. Tinción HE; 100X; (B) núcleos desplazados a la periferia del citoplasma $(\uparrow)$. Tinción HE; 400X. en diferentes áreas (Figura 2a), con remodelación tisular por tejido conectivo y presencia de megacariocitos, lo que sugiere hematopoyesis extramedular (Figura 2b) y el hígado, infiltración de plasmocitos específicamente a nivel de las triadas portales y áreas centrolobulillares (Figura 3).

El diagnostico definitivo fue: plasmocitoma.
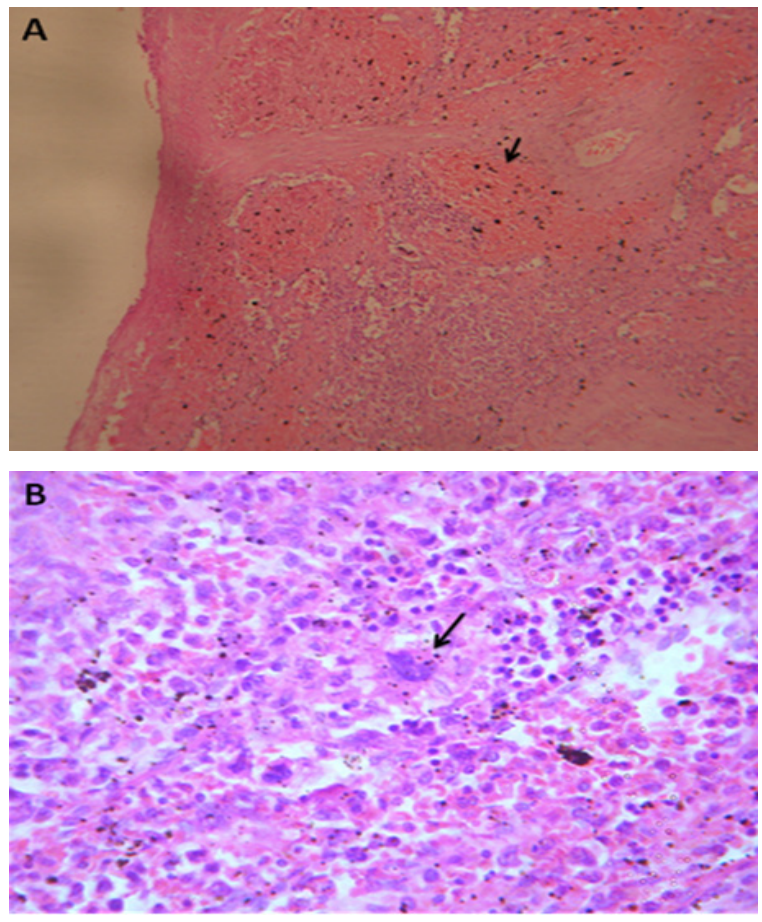

Figura 2. Corte histológico de bazo: (A) focos hemorrágicos $(\uparrow)$. Tinción HE; 100X. (B) presencia de un megacariocito $(\uparrow)$.Tinción $\mathrm{HE} ; 400 \mathrm{X}$.

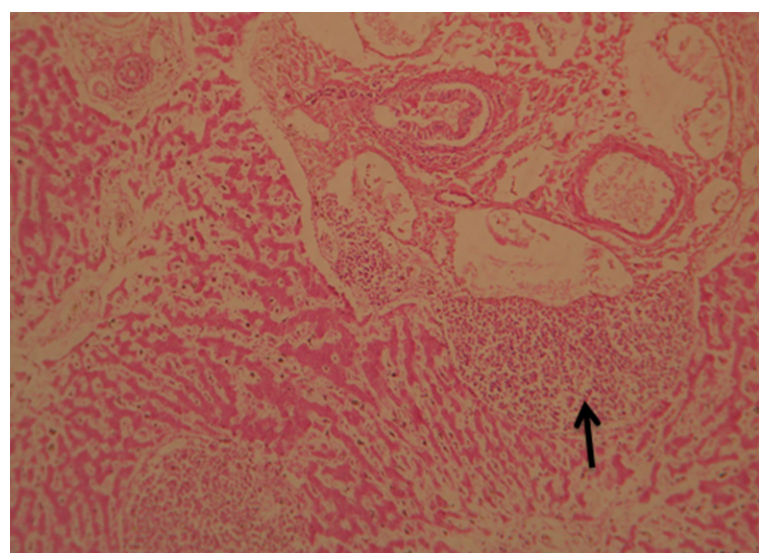

Figura 3. Corte histológico de hígado: (triada portal), infiltración de plasmocitos $(\uparrow)$. Tinción HE; 100X. 


\section{DISCUSION}

Al examen físico del paciente se evidenció síndrome ascítico por hipoproteinemia, indicando un diagnostico presuntivo de una hepatopatía por los hallazgos adicionales de una bilirrubinuria marcada; sin embargo el edema frio en el miembro posterior izquierdo, era independiente al síndrome ascítico, considerándose un linfaedema secundario (17).

Los hallazgos hematológicos, se observó un proceso inflamatorio severo, considerándose una respuesta inefectiva e inapropiada, por la presentación de una desviación a la izquierda degenerativa (18).

Los plasmocitomas extramedulares generalmente, son tumores solitarios y benignos (19) y algunas veces pueden ocasionar metástasis a nódulos linfáticos (20). En el presente caso, se encontró metástasis a hígado.
Los plasmocitomas extramedulares primarios en nódulos, han sido reportados en humanos y son considerados raros $(20,21)$ , en los autores no encontraron referencias bibliográficas que reporten la presentación de este caso especifico de neoplasia en canino. La escisión quirúrgica está indicada en los casos de plasmocitomas extramedulares localizados y únicos (9), pero cuando se encuentran en la mayoría de los nódulos linfáticos, como en este caso, la quimioterapia es la indicada, con la probabilidad de recidivas (22).

Este tipo de neoplasia adquiere especial importancia en la especie canina, ya que los hallazgos del examen físico y hemático podrían llegar a formular un pre-diagnostico o diagnostico definitivo de linfoma, neoplasia de mayor frecuencia de presentación y que en muchas ocasiones llevaría a decidir la eutanasia del paciente.

\section{REFERENCIAS}

1. Fink-Puches R, P Zenahlik, et al. "Primary cutaneous lymphomas: applicability of current classification schemes (European Organization for Research and Treatment of Cancer, World Health Organization) based on clinicopathologic features observed in a large group of patients." Blood 2002, 99(3):800.

2. Ocaña Cristeta F. Skin neoplasias in dogs and cats. Prof Vet (España), 2007; 166 (67): $10-16$.

3. Cangul, I., M. Wijnen, et al. "Clinicopathological aspects of canine cutaneous and mucocutaneous plasmacytomas." ] Vet Med A 2002; 49(6):307-312.

4. Choi YK, Lee JY, Kim DY, Park JI, Jeong SW, Park HM, Park C. Uterine extramedullary plasmacytoma in a dog. Vet Rec 2004; 154(22):699-700.

5. Weigt A, M McCracken, et al. "Extramedullary Plasmacytoma in the Canine Trachea: Case Report and Literature Review." Comp Cont Ed Pract Vet 2001; 23(2):143-156.

6. Hayes AM, SP Gregory, S Murphy, JF MCConnell, JC Patterson-Kane. Solitary extramedullary plasmacytoma of the canine larynx. J Small Anim Pract 2007; 48(5):288-291.
7. Michau T, D Proulx, et al. Intraocular extramedullary plasmacytoma in a cat. Vet Ophthalmol 2003, 6(2):177-181.

8. Kupanoff PA, Popovitch CA, Goldschmidt MH. Colorectal plasmacytomas: a retrospective study of nine dogs. J Am Anim Hosp Assoc 2006; 42(1):37-43.

9. Rannou B, P Hélie, et al. Rectal plasmacytoma containing hemosiderin in a dog. Vet Pathol 2009, 46:1181.

10. Majzoub M, W Breuer, et al. Histopathologic and immunophenotypic characterization of extramedullary plasmacytomas in nine cats. Vet Pathol 2003; 40(3):249-253.

11. Ramos-Vara J, M Miller, et al. Immunohistochemical detection of multiple myeloma 1/interferon regulatory factor 4 (MUM1/IRF-4) in canine plasmacytoma: comparison with CD79a and CD20. Vet Pathol 2007; 44(6):875.

12. Vail DM, Thamm DH. Tumores hematopoyeticos. En: Tratado de Medicina Interna Veterinaria. Stephen Ettinger, Edward Feldman (Eds). España, ELSEVIER, 2007. 
13. Mayer M, Kerr M, Grier C, MacDonald V. Immunoglobulin A multiple myeloma with cutaneous involvement in a dog. Can Vet J 2008; 49(7):694-702.

14. Dores GM, Landgren O, McGlynn KA, Curtis RE, Linet MS, Devesa SS. Plasmacytoma of bone, extramedullary plasmacytoma, and multiple myeloma: incidence and survival in the United States, 1992-2004. Br J Haematol 2009;144(1):86-94.

15. Sternberg R, Wypij J, Barger AM. Extramedullary and solitary osseous plasmacytomas in dogs and cats. Urbana 2009;51:61802.

16. Ware K, Gieger T. Use of strontium 90 plesiotherapy for the treatment of a lingual plasmacytoma in a dog. J Small Anim Pract 2011;52(4):220-3.

17. Kerchner K, Fleischer A, Yosipovitch G. Lower extremity lymphedema:: Update: Pathophysiology, diagnosis, and treatment guidelines. J Am Acad Dermatol 2008;59(2):324-31.

18. Stockham SL, Keeton KS, Szladovits B. Clinical assessment of leukocytosis: distinguishing leukocytoses caused by inflammatory, glucocorticoid, physiologic, and leukemic disorders or conditions. Vet Clin North Am Small Anim Pract 2003;33(6):1335 - 57
19. Wright ZM, Rogers KS, Mansell J. Survival data for canine oral extramedullary plasmacytomas: a retrospective analysis (1996-2006). J Am Anim Hosp Assoc 2008; 44(2):75-81.

20. Lim YH, Park SK, Oh HS, Choi JH, Ahn MJ, Lee YY, Kim IS. A case of primary plasmacytoma of lymph nodes. Korean J Intern Med 2005; 20(2):183-6.

21. Yoshida A, S Borkar, et al. Incidental Detection of Concurrent Extramedullary Plasmacytoma and Amyloidoma of the Nasopharynx on [18F] Fluorodeoxyglucose Positron Emission Tomography/Computed Tomography. J Clin Oncol 2008; 26(35):5817.

22. Radhakrishnan A, R Risbon, et al. Progression of a solitary, malignant cutaneous plasmacell tumour to multiple myeloma in a cat. Vet Comp Oncol 2004; 2(1):36-42. 